\title{
Prediction of column leaching behaviour based on batch leaching tests with different liquid to solid ratios
}

\author{
Jiajie Tang ${ }^{\text {i) }}$, Hirofumi Sakanakura ${ }^{\text {ii) }}$, Mikako Nakagawa ${ }^{\text {ii) }}$, Atsushi Takai ${ }^{\text {i) }}$, and Takeshi Katsumi ${ }^{\text {i) }}$ \\ i) Graduate School of Global Environmental Studies, Kyoto University, Yoshida-Honmachi, Sakyo-ku, Kyoto, 606-8501, Japan \\ ii) Center for Material Cycles and Waste Management Research, National Institute for Environmental Studies, 16-2 Onogawa, Tsukuba, \\ 305-0053 Japan
}

\begin{abstract}
Batch leaching tests with different liquid per solid ratios and contact times were conducted on tsunami deposit soil. The chemicals of concern are arsenic (As), boron (B), and sodium (Na). Two parameters were obtained from the tests, which are the partition coefficient $\left(K_{\mathrm{d}}\right)$ and the available mass of contaminants per unit mass of soil $\left(M_{\mathrm{T}}\right)$. Here, the "available mass of contaminants" refers to the mass of contaminants that will take part in adsorption-desorption reaction, but not the total mass of contaminants which are present in the soil system. Using these two parameters, temporal concentration profiles based on the analytical solution of a one-dimensional advection-dispersion equation were plotted for different contact time, and compared with the results of column leaching tests, which were conducted separately using $10-\mathrm{cm}$ long and $5-\mathrm{cm}$ high columns. From the comparisons, multiple sets of two parameters at different contact times are needed to express the column test results for all three chemicals. To predict the column leaching behaviour from batch test with minimum numbers of contact times, two contact times, 6 and/or 24 hours can be selected.
\end{abstract}

Keywords: advection-dispersion curve, batch leaching test, column test, naturally contaminated soil

\section{INTRODUCTION}

Various cases of different kinds of toxic elements from geogenic processes have been found in excavated materials, in many countries (Cremisini and Armiento, 2016; Li et al., 2014; Li et al., 2018). In Japan, the soils containing natural contamination are frequently found to exceed the limits of leaching values regulated by the Soil Contamination Countermeasures Law (SCCL). As a result, it has become a serious issue in Japan to handle such materials, and some of the materials with low hazard are recommended to be reused for the other purposes of construction (Katsumi et al., 2018). Excavated soils containing natural contaminants should be properly stored/reused in a manner which prevents adverse environmental impacts due to leaching and migration of contaminants. To verify that the environmental impacts are negligible or within acceptable levels, geochemical composition, leaching concentrations, and transport of contaminants in the subsurface have become important issues to consider among other geoenvironmental parameters.

Because total content can be a poor indicator of environmental by such materials, leaching tests have become increasingly important for assessing the risks of release of potential contaminants. In Japan batch leaching test is applied according to the Japanese
Notification No. 46 (JLT-46), where water is brought into contact with the solid materials and leaching concentrations is analysed. In general, batch leaching tests offers better reproducibility and has a simplistic design (Jakson et al., 1984). However, the shaking process included in JLT-46 might artificially cause the change of leaching behaviour because of abrasion of particles, destruction of aggregates, mobilization of colloids, and so on (Grathwohl, 2009). In addition, its applicability to the soils with natural contamination is questionable because it does not consider the natural condition of the soil (e.g. compacted state, water content), conditions of the surface environment (e.g. temperature), and flow conditions (Katsumi, 2017).

Over recent years, the column percolation test under saturated/unsaturated flow conditions has been more widely applied, because it can more closely represent the site conditions (Grathwohl, 2007; Inui et al., 2018; Rennert et al., 2010). For example, it can closely simulate in-situ water in contact with the solid materials and allow the monitoring of leaching behavior with time (Roussat et al., 2008). However, this method generally has many challenges including installation of a column, need for well-functioning equipment, skilled techniques, operating conditions, and time-consuming.

Sakanakura and Ito (2018) proposed the concept of 
applying the available mass of contaminants per unit mass of soil $\left(M_{\mathrm{T}}\right)$ and the partition coefficient $\left(K_{\mathrm{d}}\right)$, to evaluate the release of trace elements, the parameters can be obtained from batch tests with different liquid/solid ratio $(L / S)$. Following Sakanakura and Ito (2018), this study applied these two parameters to plot temporal concentration profiles based on the analytical solution of a one-dimensional advection-dispersion equation. The leaching profiles arsenic (As), boron (B), and sodium $(\mathrm{Na})$ are compared with the results of column leaching tests, which were conducted separately using 10-cm long and 5-cm high columns.

\section{MATERIALS AND METHODS}

\subsection{Material}

Tsunami deposit soil generated from the 2011 Tohoku earthquake, was collected from Kesennuma city and used after air-drying $(w=0.6 \%)$, as a representative of naturally contaminated soils planned to be used in embankment and/or other shallow geo-structures. It has particle density of $2.65 \mathrm{~g} / \mathrm{cm}^{3}$, and its particle size distribution determined according to JGS 0131-2000 is shown in Fig. 1.

\subsection{Batch leaching test}

To determine the leaching amount of elements in the water after contacting the soil, a series batch leaching test was carried out with for twenty-five cases with different $L / S$ ratio of $1,3,10,100$, and $300 \mathrm{~L} / \mathrm{kg}$, and contact time $\left(T_{\mathrm{c}}\right)$ of $0.2,6,24,168$, and $672 \mathrm{~h}$.

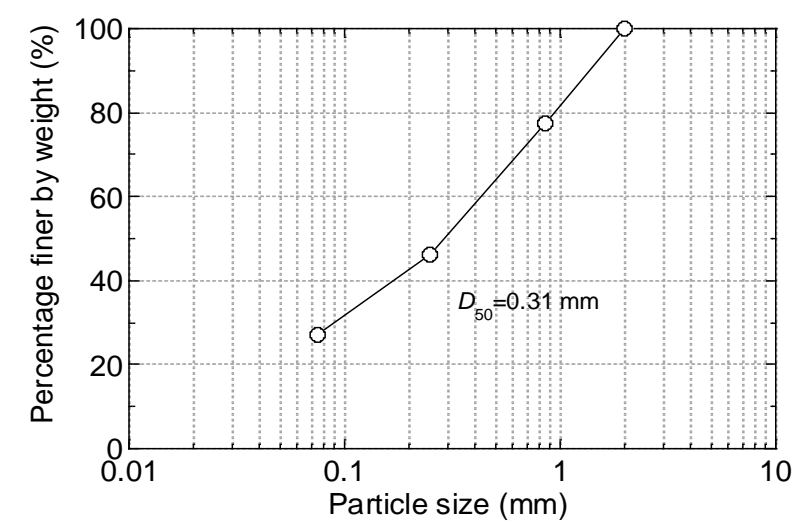

Fig. 1. Particle size distribution of sand from tsunami deposit.

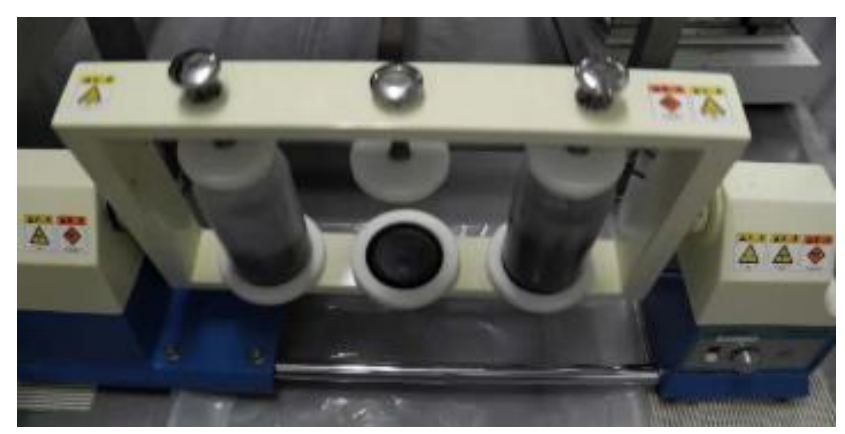

Fig. 2. Rotation device used for batch leaching test.
Soil sample was added into plastic bottles together with $100 \mathrm{~mL}$ deionized water to achieve the desired $L / S$ ratio. Bottles were placed into the end-over-end rotation machine, shown in Fig. 2, and rotated at $3.5 \mathrm{rpm}$ for desired $T_{\mathrm{c}}$. After the rotation process, the bottles were left standing between 10-30 minutes, and thereafter supernatant was filtered using a $0.45 \mu \mathrm{m}$ membrane filter. Properties of the leachate including $\mathrm{pH}, \mathrm{EC}$, and As, B, Na, and concentrations of other water-soluble elements were analysed by inductively coupled plasma atomic emission spectroscopy (ICP-OES) and inductively coupled plasma mass spectrometry (ICP-MS).

\subsection{Column percolation test}

Soil sample was poured (without compaction) into $10-\mathrm{cm}$ long and $5-\mathrm{cm}$ high acrylic column, to achieve a packing density of $1.47 \mathrm{~g} / \mathrm{cm}^{3}$ and void ratio $(e)$ of 0.76 . By employing a peristaltic pump, deionized water was introduced to the column from the bottom, at a flow rate of $12 \mathrm{~mL} / \mathrm{h}$, to saturate the soil porous medium. After the saturation process, permeation was continued for one column as equilibration time $\left(T_{\mathrm{e}}\right)$ was 0 days, but for the second column, the water flow was stopped until $T_{\mathrm{e}}=10$ days. Leachate was collected at regular time intervals until reaching a cumulative $L / S$ ratio of $8 \mathrm{~L} / \mathrm{kg}$. Properties of the leachate were also in a similar way to batch test.

\section{ANALYSIS}

\subsection{1-D advection-dispersion analytical solution}

Transport of contaminants in soils can be modelled by a one-dimensional form of the advection-dispersion (AD) equation (1) proposed by Miller and Webber (1986), where $C$ is solute concentration, $v$ is the average linear flow velocity, $z$ is the spatial domain, $t$ is time, and $D$ is a constant dispersion coefficient, $n$ is porosity of soil. Given the boundary conditions in equation (2), an analytical solution as equation (3) can be derived.

$$
\left.\begin{array}{c}
\frac{\partial C}{\partial t}=-v \frac{\partial C}{\partial z}+D \frac{\partial^{2} C}{\partial z^{2}} \frac{1-n}{n} \\
C=C_{0}(t=0) \\
C=0(t \rightarrow \infty)
\end{array}\right\} \text { Boundary conditons }
$$

$$
\begin{aligned}
C(t)=C_{0}-\frac{1}{2} C_{0}[ & \operatorname{erfc}\left(\frac{R H-v t}{2 \sqrt{D R t}}\right)+ \\
& \left.+\exp \left(\frac{v H}{D}\right) \operatorname{erfc}\left(\frac{R H+v t}{2 \sqrt{D R t}}\right)\right]
\end{aligned}
$$

where $C(\mathrm{mg} / \mathrm{L})$ is the leaching concentration for a given time $(t), C_{0}(\mathrm{mg} / \mathrm{L})$ is the initial leaching concentration, $H(\mathrm{~m})$ is the height of soil column, $t(\mathrm{~s})$ is time, $v(\mathrm{~m} / \mathrm{s})$ is velocity of water flow, $D\left(\mathrm{~m}^{2} / \mathrm{s}\right)$ is the dispersion coefficient, and $R(-)$ is the retardation factor which is calculated from equation (4). 


$$
R=1+\frac{\rho_{\mathrm{d}}}{n} K_{\mathrm{d}}
$$

where $\rho_{\mathrm{d}}\left(\mathrm{g} / \mathrm{cm}^{3}\right)$ is the dry density of soil, $n(-)$ is the porosity of soil, $K_{\mathrm{d}}(\mathrm{L} / \mathrm{kg})$ is a partition coefficient.

\subsection{Transport parameters from batch leaching test}

In equation (4), $K_{\mathrm{d}}$ as a partition coefficient can be estimated from the Henry isotherm equation (5).

$$
q=K_{\mathrm{d}} C_{\mathrm{r}}
$$

where $q(\mathrm{mg} / \mathrm{kg})$ is adsorption capacity per unit mass of soil, $C_{\mathrm{r}}(\mathrm{mg} / \mathrm{L})$ is contaminant concentration in solution for a given $L / S$ ratio.

Partition coefficient $\left(K_{\mathrm{d}}\right)$ and the available mass of contaminants per unit mass of soil $\left(M_{\mathrm{T}}\right)$ were used to plot the temporal concentration profiles based on the analytical solution of a one-dimensional $\mathrm{AD}$ equation. As illustrated in Fig. 3, the "available mass of contaminants" refers to the mass of contaminants that take part in the adsorption-desorption process, but not the total mass of contaminants that are present in the soil system.

For a given time, $M_{\mathrm{T}}$ can be assumed to be the sum of contaminants adsorbed at the particle surface $(q)$ and the contaminants in solution $\left(M_{\mathrm{L}}\right)$, as equation (6) (Sakanakura and Ito, 2018). With the calculation in equation (5), equation (6) can transfer to equation (7).

$$
\begin{gathered}
q+M_{\mathrm{L}}=M_{\mathrm{T}} \\
K_{\mathrm{d}} C_{\mathrm{r}}+M_{\mathrm{L}}=M_{\mathrm{T}}
\end{gathered}
$$

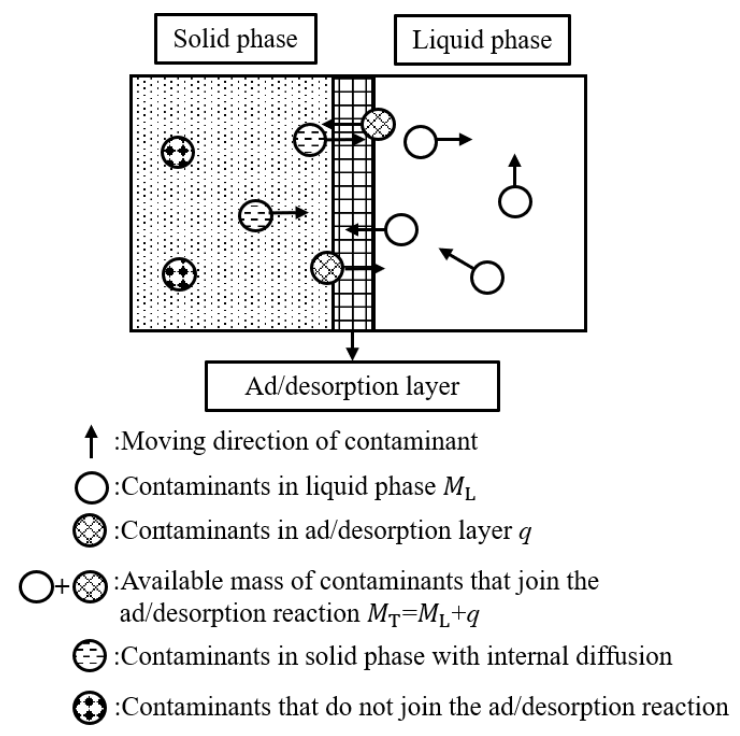

Fig. 3. Schematical view of contaminants in soil-water system.
The relationship of $C_{\mathrm{r}}(\mathrm{mg} / \mathrm{L})$ and $M_{\mathrm{L}}(\mathrm{mg} / \mathrm{kg})$ can be obtained from batch test. For a given $L / S$ ratio, $M_{\mathrm{L}}$ $(\mathrm{mg} / \mathrm{kg})$ can be calculated using equation (8).

$$
M_{\mathrm{L}}=C_{\mathrm{r}} \times \frac{L}{S}
$$

where $L$ (litres) is the volume of water, $S(\mathrm{~kg})$ is soil mass.

As shown in Fig. 4, a linear fit can be used to describe the relationship between $M_{\mathrm{L}}$ and $C_{\mathrm{r}}$ obtained from the batch test, for a particular contact time. In this case, $M_{\mathrm{T}}(\mathrm{mg} / \mathrm{kg})$ is assumed to be the intercept on the vertical axis, while $K_{\mathrm{d}}(\mathrm{L} / \mathrm{kg})$ is the slope of the linear function. The calculation process can be shown in Fig. 4.

Assuming the boundary conditions $\left(C=C_{0}\right.$ at $\left.t=0\right)$ indicated in equation (2) are satisfied and leaching value is negligible (assume $M_{\mathrm{L}} \approx 0$ ), equation (7) can be modified to equation (9), so as to estimate $C_{0}$.

$$
C_{0}=\frac{M_{\mathrm{T}}}{K_{\mathrm{d}}}
$$

The AD curve using batch test data of different contact time can be plotted as shown in Fig. 5. To avoid over/underestimation of leaching concentrations, the $\mathrm{AD}$ curve was plotted using parameters estimated for different contact times, depending on the time scale of interest. For example, for batch test data when $T_{\mathrm{c}}=t_{1}$, the $\mathrm{AD}$ curve starts with a $C_{0}=C_{0-\mathrm{I}}$, when $T_{\mathrm{c}}=t_{2}, \mathrm{AD}$ curve starts with a $C_{0}=C_{0-\text {-II }}$. Since the values of $C_{0}$ $(\mathrm{mg} / \mathrm{L})$ and $R(-)$ vary for different contact time (indicated in Table 1), an AD curve using different contact time data with a concentration peak was drawn as schematically shown in Fig. 5. For time period I that near $t_{1}$, the data set of $t_{1}$ is used; for time period II that near $t_{2}$, the data set corresponding to $t_{2}$ is used for the calculation.

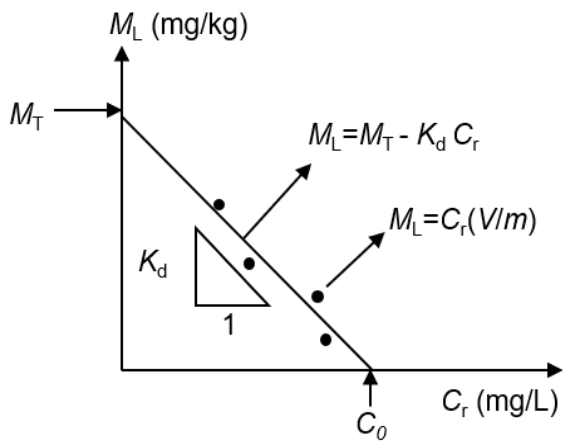

Fig. 4. Relationship between $C_{\mathrm{r}}$ and $M_{\mathrm{L}}$ using Henry isotherm. 


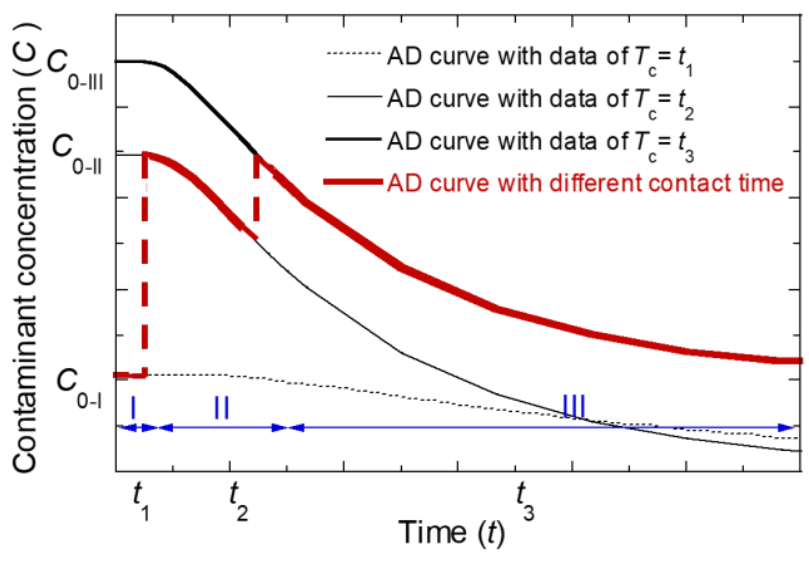

Fig. 5. AD curves from batch test with different contact time.

\section{RESULTS AND DISCUSSION}

For the calculation result from batch data, a longer contact time has a higher $C_{0}(\mathrm{mg} / \mathrm{L})$ and a smaller $R(-)$. In addition, the simulation curve sometimes might show a peak (or shift) in the left side of the figure, and the ad/desorption parameters change the value, as indicated in Table 1.

As shown in Fig. 6 to 8, the temporal concentration profiles of $\mathrm{As}, \mathrm{B}$, and $\mathrm{Na}$ were plotted and are compared with the data from column tests. The concentration profiles can be considered as a type of elution curve. Martin-Lara et al. (2012) pointed out that there commonly is an increasing trend of metal concentration which is related to the breakthrough curve but referred to as the desorption state. It normally has an unsymmetrical frequency distribution shape, and a flatter decrease curve follows the rapid increase.

\subsection{Arsenic (As)}

In Fig.6, the $\mathrm{AD}$ curves showed very low As leaching concentrations that are below the acceptable levels under SCCL. Comparing with column test results, the profiles showed an underestimation of As leaching. One of the reasons might because of the little/no-flow condition in the batch test. Nikoladis et al. (2003) found out that leaching of As in the column test was promoted by a high flow rate. However, from our test method, as the rotation speed is relatively low, it is difficult to achieve a high water flow even the water flow is assumed to be negligible. This phenomenon also points out the limitation of batch leaching test, whereby higher concentrations of As might be leached during the service life of surface geostructures constructed using naturally contaminated soils.

Comparing the leaching profiles from the two column tests, it was found that the column allowed to saturate for 10 days had higher leaching concentration, which initially was about 4 times higher but the differences became smaller with more flow. This might be the evidence that the internal diffusion occurs during the equilibration time, and more As diffused to the soil surface layer and took part in the ad/desorption reactions.

\subsection{Boron (B)}

As shown in Fig. 7, differences in the B leaching concentration in the two columns were observed mainly in the first $48 \mathrm{~h}$, where B was slightly higher in the soil column saturated for 10 days. Majidi et al. (2010) found that B is mainly adsorbed at the outer-sphere complex in the soil samples. Therefore, for the column without an equilibration time, B on the soil surface was washed out by the water flow. On the other hand, for the column with a 10-days equilibration time, surface $\mathrm{ad} /$ desorption can reach a relative equilibrium state, which causes the leaching of B to be milder in the first $48 \mathrm{~h}$.

For the AD curves, due to the lower limit of ICP measurement, the concentration of $0.2 \mathrm{~h}$ contact time is not shown herein. By comparing with the simulation data and experimental data, a contact time of 6 or $24 \mathrm{~h}$ can better fit the data than others. However, the experimental data is higher than the calculation result from the batch test, which might indicate that the internal diffusion occurred, and the ad/desorption reaction might stay for a relative equilibrium state later.

\subsection{Sodium $(\mathrm{Na})$}

As shown in Fig. 8, Na concentrations from the two columns did not vary, only in the initial period where the column with 10-days equilibration period had a slightly higher $\mathrm{Na}$ concentration, which reached 300 $\mathrm{mg} / \mathrm{L}$. This might indicate that the leaching of $\mathrm{Na}$ has no direct relationship with equilibration time.

Moreover, for the AD curve with different contact times, the concentration of $\mathrm{Na}$ shows a peak between $0.2 \mathrm{~h}$ and $6 \mathrm{~h}$. Because the value of $C_{0}$ has a rapid increase, and $R$ has a rapid decrease with the contact time change, as indicated in Table 1.

Table 1. AD fitting parameters from batch tests. (ND here refers to "not detected")

\begin{tabular}{|c|c|c|c|c|c|c|c|c|c|c|c|c|}
\hline \multirow{2}{*}{$\begin{array}{l}\text { Contact time, } \\
T_{\mathrm{c}}(\mathrm{h})\end{array}$} & \multicolumn{4}{|c|}{ Arsenic (As) } & \multicolumn{4}{|c|}{ Boron (B) } & \multicolumn{4}{|c|}{ Sodium $(\mathrm{Na})$} \\
\hline & $\begin{array}{l}M_{\mathrm{T}} \\
(\mathrm{mg} / \mathrm{kg})\end{array}$ & $\begin{array}{l}K_{\mathrm{d}} \\
(\mathrm{L} / \mathrm{kg})\end{array}$ & $\begin{array}{l}C_{0} \\
(\mathrm{mg} / \mathrm{L})\end{array}$ & $R(-)$ & \begin{tabular}{|l}
$M_{\mathrm{T}}$ \\
$(\mathrm{mg} / \mathrm{kg})$
\end{tabular} & $\begin{array}{l}K_{\mathrm{d}} \\
(\mathrm{L} / \mathrm{kg})\end{array}$ & $\begin{array}{l}C_{0} \\
(\mathrm{mg} / \mathrm{L})\end{array}$ & $R(-)$ & \begin{tabular}{|l}
$M_{\mathrm{T}}$ \\
$(\mathrm{mg} / \mathrm{kg})$
\end{tabular} & $\begin{array}{l}K_{\mathrm{d}} \\
(\mathrm{L} / \mathrm{kg})\end{array}$ & $\begin{array}{l}C_{0} \\
(\mathrm{mg} / \mathrm{L})\end{array}$ & $R(-)$ \\
\hline 0.2 & 0.04 & 12.1 & 0.003 & 42.1 & ND & ND & ND & ND & 198 & 1.97 & 106 & 7.34 \\
\hline 6 & 0.17 & 29.4 & 0.005 & 100 & 0.36 & 1.72 & 0.22 & 6.86 & 240 & 0.69 & 347 & 3.34 \\
\hline 24 & 0.23 & 36.7 & 0.006 & 125 & 0.59 & 2.34 & 0.25 & 8.94 & 226 & 0.65 & 349 & 3.19 \\
\hline 168 & 0.40 & 83.2 & 0.004 & 283 & 0.72 & 2.08 & 0.36 & 8.08 & 240 & 0.59 & 417 & 2.96 \\
\hline 672 & 0.51 & 119 & 0.004 & 405 & 0.72 & 1.81 & 0.41 & 7.13 & 270 & 0.55 & 495 & 2.85 \\
\hline
\end{tabular}




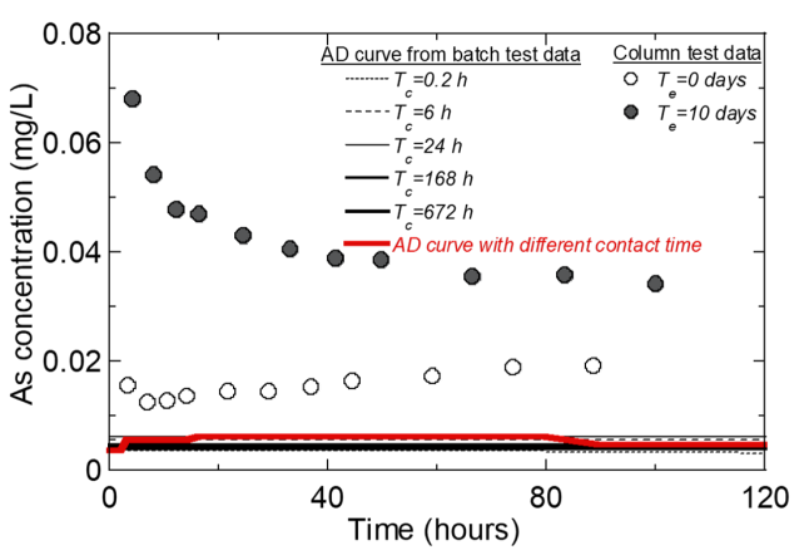

Fig. 6. Leaching behaviour of arsenic.

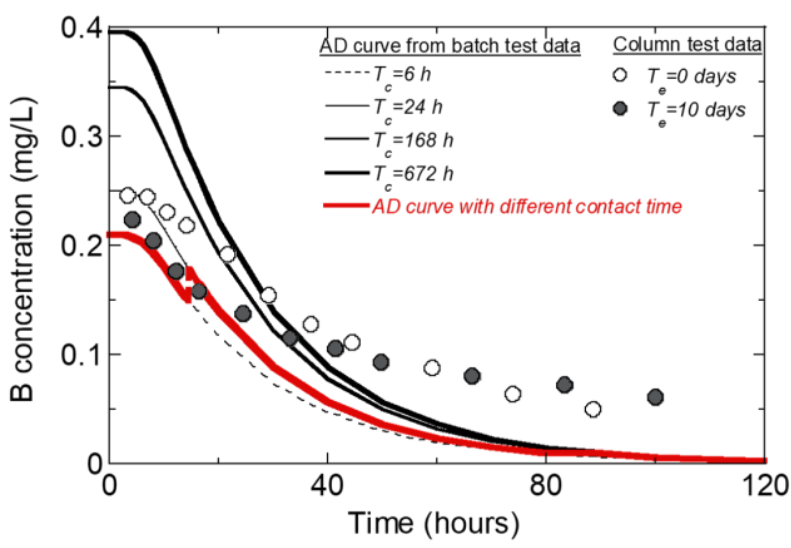

Fig. 7. Leaching behaviour of boron.

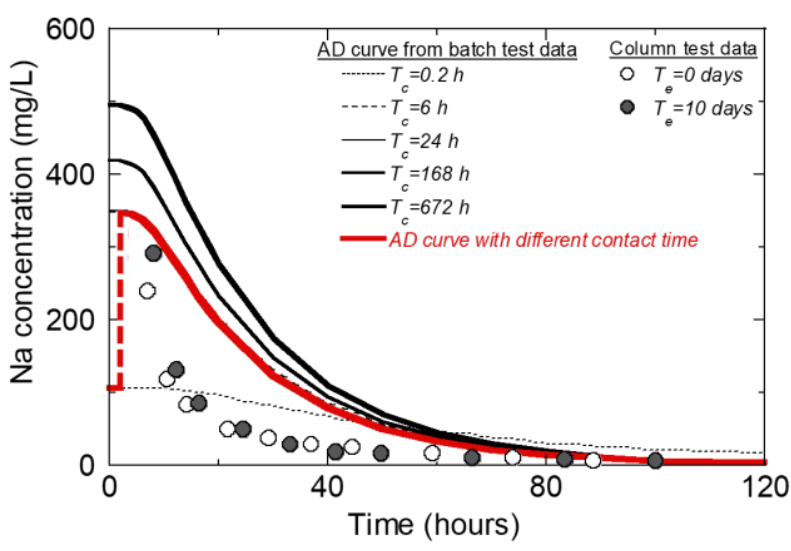

Fig. 8. Leaching behaviour of sodium.

\section{CONCLUSIONS}

Ad/desorption parameters $\left(K_{\mathrm{d}}\right.$ and $\left.M_{\mathrm{T}}\right)$ obtained from batch test can predict the leaching behaviour of contaminants (in a similar way to column tests) using the one-dimensional prediction model. However, this model still has its limitations especially when predicting the leaching behaviour of As.

Comparing the data fitting of $\mathrm{B}$ and $\mathrm{Na}$, although the related parameters change with the contact time, 6 and/or $24 \mathrm{~h}$ should be considered when simply estimating the ad/desorption parameters. However, as the parameters change with the contact time, a peak (shift) might present in the $\mathrm{AD}$ curve with different contact time.

In addition, the comparison of two column tests also shows the internal diffusion and the ad/desorption equilibrium with/without an equilibration time. Besides, the comparison of the $\mathrm{AD}$ curves and actual experiment data of As indicates that the limitation of the current batch leaching test.

In the future, the prediction method will be extended to fit more ad/desorption model and improve the accuracy of column test concentration simulation, to provide better for evaluating the long-term stability of the soil sample.

\section{ACKNOWLEDGEMENTS}

Sincere appriciations to Mr. M. Hirayama, Dr. S. Back and Dr. H. Ishimori (National Institute for Environmental Studies), L. W. Gathuka (Kyoto University) for their great assistance.

\section{REFERENCES}

1) Cremisini, C. and Armiento, G. (2016): High geochemical background of potentially harmful elements. The "geochemical risk" and "natural contamination" of soils and water: awareness and policy approach in Europe with a focus on Italy. Rendiconti Lincei-Scienze Fisiche E Naturali, 27(1): 7-20.

2) Grathwohl, P. and Sloot V. (2007): Chapter 5.6. Groundwater Risk Assessment at Contaminated Sites (GRACOS): Test Methods and Modelling Approaches. Groundwater Science and Policy, 291-315. doi: 10.1039/9781847558039-0029

3) Grathwohl, P. and Susset, B. (2009): Comparison of percolation to batch and sequential leaching tests: Theory and data. Waste Management, 29(10), 2681-2688. doi: 10.1016/j.wasman.2009.05.01

4) Inui, T., Hori, M., Takai, A., and Katsumi, T. (2018): Column Percolation Tests for Evaluating the Leaching Behavior of Marine Sediment Containing Non-anthropogenic Arsenic. Proceedings of the 8th International Congress on Environmental Geotechnics Volume 1, Hangzhou, China.

5) Jackson, D. R., Garrett, B. C., and Bishop, T. A. (1984): Comparison of batch and column methods for assessing leachability of hazardous waste. Environmental Science \& Technology, 18(9), 668-673. doi: 10.1021/es00127a007

6) Japanese Geotechnical Society (2014): The Guidelines for Effective Use of Reconstruction Materials Recovered from Disaster Waste. Retrieved from https://www.jiban.or.jp/ images/file/Guidelines_all.pdf

7) Japanese Ministry of Environment (2003): Matters for determining measurement methods for soil content surveys

8) Japanese Ministry of Environment (2002): Soil Contamination Countermeasures Act.

9) Kalbe, U., Berger, W., Simon, F.-G., Eckardt, J., and Christoph, G. (2007): Results of interlaboratory comparisons of column percolation tests. Journal of Hazardous Materials, 148(3), 714-720. doi: 10.1016/j.jhazmat.2007.03.039

10) Katsumi, T. (2017). Use of Excavated Soils with Natural Contamination. Geotechnical Engineering Magazine, 65(11), $1-3$.

11) Katsumi, T., Inui, T., Yasutaka, T., and Takai, A. (2018): Towards Sustainable Soil Management - Reuse of Excavated 
Soils with Natural Contamination. Proceedings of the 8th International Congress on Environmental Geotechnics Volume 1, Hangzhou, China.

12) Lager, T., Delay, M., Karius, V., Hamer, K., Frimmel, F. H., and Schulz, H. D. (2006): Determination and quantification of the release of inorganic contaminants from municipal waste incineration ash. Acta Hydrochimica et Hydrobiologica, 34, 73-85.

13) Li, J., Kosugi, T., Riya, S., Hashimoto, Y., Hou, H., Terada, A., and Hosomi, M. (2018): Pollution potential leaching index as a tool to assess water leaching risk of arsenic in excavated urban soils. Ecotoxicol Environ Saf, 147: 72-79.

14) Li, J., Wei, Y., Zhao, L., Zhang, J., Shangguan, Y., Li, F., and Hou, H. (2014): Bioaccessibility of antimony and arsenic in highly polluted soils of the mine area and health risk assessment associated with oral ingestion exposure. Ecotoxicology and Environmental Safety, 110: 308-315.

15) Majidi, A., Rahnemaie, R., Hassani, A., and Malakouti, M. J. (2010): Adsorption and desorption processes of boron in calcareous soils. Chemosphere, 80(7), 733-739. doi: 10.1016/j.chemosphere.2010.05.025

16) Martín-Lara, M., Blázquez, G., Ronda, A., Rodríguez, I., and Calero, M. (2012): Multiple biosorption-desorption cycles in a fixed-bed column for $\mathrm{Pb}(\mathrm{II})$ removal by acid-treated olive stone. Journal of Industrial and Engineering Chemistry, 18(3), 1006-1012. doi: 10.1016/j.jiec.2011.11.150
17) Miller, C. T. and Weber, W. J. (1986): Sorption of hydrophobic organic pollutants in saturated soil systems. Journal of Contaminant Hydrology, 1(1-2), 243-261. doi: 10.1016/0169-7722(86)90019-7.

18) Nikolaidis, N. P., Dobbs, G. M., Chen, J., and Lackovic, J. A. (2004): Arsenic mobility in contaminated lake sediments. Environmental Pollution, 129(3), 479-487. doi: 10.1016/ j.envpol.2003.11.005

19) Rennert, T., Meißner, S., Rinklebe, J., and Totsche, K. U. (2009): Dissolved Inorganic Contaminants in a Floodplain Soil: Comparison of In Situ Soil Solutions and Laboratory Methods. Water, Air, \& Soil Pollution, 209(1-4), 489-500. doi: 10.1007/s11270-009-0217-3

20) Roussat, N., Méhu, J., Abdelghafour, M., and Brula, P. (2008): Leaching behaviour of hazardous demolition waste. Waste Management, 28(11), 2032-2040. doi: 10.1016/j.wasman.2007.10.019

21) Sakanakura H. and Ito K. (2018): Calculation method of adsorption/desorption parameters for pollutants-holding materials by liquid-solid ratio batch test. 53rd Annual Conference of the Japanese Geotechnical Society

22) Schumer, R., Benson, D. A., Meerschaert, M. M., and Wheatcraft, S. W. (2001): Eulerian derivation of the fractional advection-dispersion equation. Journal of Contaminant Hydrology, 48(1-2), 69-88. doi: 10.1016/s0169-7722(00)00170-4 